

$$
\operatorname{Re}\left\{\frac{z f^{\prime \prime}(z)}{f^{\prime}(z)}+1\right\}>0,(z \in U)
$$

(Soodman, 1983; p.111).

Many properties or conditions for these classes of:functions are established and written as differential inequalities. For example, Mocanu(2004) established the following sharp starlikeness condition for functions $f(z)$, analytic in $U$, of the form $f(z)=z+a_{n+1} z^{n+1}+\ldots$

$$
\begin{aligned}
& \left|z f^{\prime \prime}(z)-\alpha\left(f^{\prime}(z)-1\right)\right|<n-\alpha \\
& \left|z f^{\prime \prime}(z)-\left(f^{\prime}(z)-\frac{f(z)}{z}\right)\right|<\frac{n(n+1-\alpha)}{n+1}
\end{aligned}
$$

where $0 \leq \alpha \leq n$.

Miller and Mocanu [1978] with some conditions on $\psi: C^{3} \rightarrow C$ showed that

$$
\left|\psi\left(f(z), z f^{\prime}(z), z^{2} f^{\prime \prime}(z)\right)\right|<1 \Rightarrow|f(z)|<1, z \in U
$$

and determined a class $(\Psi)$ of functions for which

$$
\operatorname{Re}\left\{\psi\left(f(z), z f^{\prime}(z), z^{2} f^{\prime \prime}(z)\right)\right\}>0 \Rightarrow \operatorname{Ref}(z)>0 z \in U .
$$

All these inequalities one can write in a more general form as differential subordinations. The concept of differential subordination was introduced by Miller and Mocanu [1981]. They showed that if $\Delta$ represents the unit disc in (1.6) and the right-half plane in (1.7), $\psi(r, s, t)$ is holomorphic and $g(z)$ is a conformal mapping of $U$ onto $\Delta$ such that $\psi(f(0), 0,0)=g(0)=f(0)$, then $(1.6)$ and $(1.7)$ can -be jointly written as:

$$
\left.\left.\psi\left(f(z), z f^{\prime}(z), z^{2} f^{\prime}(z)\right) z\right)\right) \prec g(z) \Rightarrow f(z) \prec g(z), \quad z \in U .
$$

Differential subordinations and applications to starlikeness(univalence) and convexity (univalence) have been considered by several authors: Miller and Mocanu (1985), Obradovic and Owa(1991), Kanas(1992), Bulboacă(2004).

Owa and Obradovic(1990) considered the subordination

$$
(1-\lambda) p(z)+\lambda z p^{\prime}(z) \prec\left[\frac{1+z}{1-z}\right]^{\gamma}\left[1-\lambda+\lambda \gamma \frac{2 z}{1-z^{2}}\right]=h(z), 0 \leq \gamma \leq 1, z \in U .
$$

and provided some conditions for starlikeness in the class $A=\left\{f(z): f(z)\right.$ is analytic in $U$, with $\left.f(0)=f^{\prime}(0)-1=0\right\}$. Inspired, principally, by this work we study a similar subordination and provide a condition for starlikeness. We have the following results.

Theorem 1: Let $\alpha$ be a fixed number in $[0,1]$. Let $f(z)$ be regular in $U$ with $f(0)=1.1 f$

$$
[f(z)]^{1-\alpha}\left[f(z)+z f^{\prime}(z)\right]^{\alpha} \prec\left[\frac{1+z}{1-z}\right]\left[\frac{2 z}{1-z^{2}}+1\right]^{\alpha}=h(z), \quad(z \in \mathrm{U}, \alpha \in[0,1] .
$$

then

$$
f(z) \prec \frac{1+z}{1-z}=q(z)
$$


$\Xi=\bar{y}_{1} z$, s the best dominant of this subordination.

Theorem 2: (A condition for starlikeness)

Let $f(z)=z+\sum_{n=2}^{\infty} a_{n} z^{n}, z \in U$, be regular in $U$ with $\frac{f(z)}{z f^{\prime}(z)} \neq 0 \forall z \in U$. Let $h(z)$ be a function regular in $U$ such that $h(0)=1$ and

$$
\operatorname{Re} h(z)>0, z \in U
$$

and either

$$
h(z) \text { is convex, }
$$

or

$$
H(z)=\frac{z h^{\prime}(z)}{h(z)} \text { is starlike. }
$$

If

$$
\alpha\left(1+\frac{z f^{\prime \prime}}{f^{\prime}(z)}\right)+(1-\alpha)\left(\frac{z f^{\prime}(z)}{f(z)}\right) \prec h(z), \alpha \in[0,1], z \in U
$$

then $f(z)$ is starlike in $U$.

\section{PROOFS OF THE RESULTS}

\section{Proof of Theorem 1}

To prove theorem 1 we need the following definitions due to Miller and Mocanu(1981)

Definition 2.1: We say $q(z) \in Q$ if $q(z)$ is regular $U$ and $\lim _{\substack{z \rightarrow \zeta \\ z \in l^{j}}} q(z)=\infty$

Definition 2. 2: Let $\Omega$ be a domain in $C$ and let $q(z) \in Q$. Define $\Psi_{n}(\Omega, q)$ to be the class of functions $\psi: C^{3} \rightarrow C$ that satisfy the following conditions:

(a) $\psi(r, s, t)$ is continuous in a domain $D \subset C^{3}$

(b) $(q(0), 0,0) \in D$ and $\psi(q(0), 0,0) \in \Omega$

(c) $\psi\left(r_{0}, s_{0}, t_{0}\right) \notin \Omega$ when $\left(r_{0}, s_{0}, t_{0}\right) \in D, r_{0}^{\prime}=q(\zeta), s_{0}=m \zeta q^{\prime}(\zeta)$ and

$\operatorname{Re}\left\{1+\frac{t_{0}}{S_{0}}\right\} \geq m \operatorname{Re}\left\{1+\frac{\xi^{\prime \prime}(\zeta)}{q^{\prime}(\zeta)}\right\}$. where $|\zeta|=1, q(\zeta)$ is finite and $m \geq n \geq 1$

Denote $\Psi_{\uparrow}(\Omega, q)$ by $\Psi(\Omega, q)$.

Definition 2.3: Let $h(z)$ be a conformal mapping of $U_{\ell}$ onto $\Omega$ and let $q(z) \in Q$. Denote by $\Psi_{n}(h, q)$ the class of functions $\psi \in \Psi_{n}(\Omega, q)=\Psi_{n}(h(U), q)$ which are holomorphic in their corresponding domains $D$ and satisfy $\psi(q(0), 0,0)=h(0)$. Write $\Psi_{1}(h, q)$ as $\Psi(h, q)$.

Lemma 1: [Miller and Mocanu , 1981, Theorem 8]: Let $\psi \cdot C^{3} \rightarrow C$ be holomorphic in a domain $D$ and let $h(z)$ be univalent in $U$. Suppose $f(z)=a+f_{n} z^{n}+\ldots$ is regular in $U$. $f(z) \neq a ; n \geq 1$, ' $\left(f(z), z f^{\prime}(z), z^{2} f^{\prime \prime}(z)\right) \in D, z \in U$ and $\psi\left(f(z), z f^{\prime}(z), z^{2} f^{\prime \prime}(z)\right) \prec h(z)$. If the differential equation $\psi\left(q(z), z q^{\prime}(z), z^{2} q^{\prime \prime}(z)\right)=h(z)$ has a univalent solution $q(z) \in Q$ with 
$q(0)=a$, and if $\psi \epsilon \Psi_{n}(h, q)$ then $f(z) \prec q(z)$ and $q(z)$ is the best dominant.

Now, let $\psi$ be such that $\psi(r, s, t)=r^{1-\alpha}[r+s]^{\alpha}$. We can rewrite (1.11) as

$$
\psi\left(f(z), z f^{\prime}(z), z^{2} f^{\prime \prime}(z)\right) \prec h(z)
$$

Applying lemma 1, we only need to show that:

(a) $q(z)=\frac{1+z}{1-z}$ is the solution of the differential equation

$$
\psi\left(q(z), z q^{\prime}(z), z^{\prime} q^{\prime \prime}(z)\right)=h(z)
$$

(b) $q(z)$ is univalent and $q(0)=f(0)$, and thát

(c) $\psi \in \Psi_{n}(h, q)$.

For the proof of (a), we solve the differential equation (2.2) which we rewrite as:

$$
[q(z)]^{1-\alpha}\left[q(z)+z q^{\prime}(z)\right]^{\alpha}=\left[\frac{1+z}{1-z}\right]\left[\frac{2 z}{1-z^{2}}+1\right]^{\alpha}=h(z), \quad(z \in U, \alpha \in[0, I]) .
$$

To solve (2.3), we use the transformation

$$
q_{l}(z)=q^{l / \alpha}(z)
$$

which enables us to rewrite (2.3) as

$$
q_{l}(z)+\alpha z q_{l}^{\prime}(z)=h^{l / \alpha}(z)
$$

-This is a first order linear differential equation in $g_{1}(z)$ with solution given by

$$
q_{1}(z)=\frac{1}{\alpha z^{1 / \alpha}} \cdot \int_{0}^{z}\left(\frac{1+t}{1-t}\right)^{1 / \alpha}\left(\frac{2 t}{1-t^{2}}\right) t^{(1 / \alpha)-1} d t
$$

Writing $w=\left(\frac{1+t}{1-t}\right)^{\frac{1}{\alpha}}$ and $s=\left(\frac{1+z}{1-z}\right)^{\frac{1}{\alpha}} z^{\frac{1}{\alpha}}$, we see that

$$
q_{1}(z)=\frac{1}{\alpha z^{1 / \alpha}} \int_{0}^{\infty} d w
$$

From which we have $q_{l}(z)=\left(\frac{1+z}{1-z}\right)^{\frac{1}{\alpha}}$ and easily obtain $q(z)=\frac{1+z}{1-z}$.

For the proof of (b), we use the definition of a univalent function to show that $q(z)=\frac{1+z}{1-z}$ is univalent. Now suppose $q\left(z_{1}\right)=q\left(z_{2}\right), z_{1}, z_{2} \in U$ then it is not difficult to see that it would imply $z_{1}=z_{2}$ Also $q(0)=1=f(0)$.

To prove (c), we show that $\left.\psi \in \Psi_{n}(h(r z), q(r z)), r \in\right] 0,1\left[\right.$ rather than $\psi \in \Psi_{n}(h, q)$ because we want to ensure that the conditions of the theorem are satisfied on $\bar{\Omega}=\overline{h(U)}$. To do this, we note that $\psi(r, s, t)=r^{1-u}[r+s]^{\prime \prime}$ is holomorphic in a domain $\mathrm{DC} \boldsymbol{C}^{3}$,

$(q(0), 0,0)=(1,0,0) \in C^{3}$, and $\psi(1,0,0) \in \Omega=\bar{U}$ and show that $\psi\left(q(r \zeta), m r \zeta q^{\prime}(r \zeta), r^{2} q\right.$ " $\left.(r \zeta)\right) \notin h_{r}(U)$, where $\left.h_{r}=h(r z), r \in\right] 0,1[$ ,$|\zeta|=1$ and $m \geq 1$. Using $[q(z)]^{1-\alpha}\left[q(z)+z q^{\prime}(z)\right]^{\alpha x}=h(z)$, we obtain 
$\psi\left(q\left(r \zeta, m r \zeta q^{\prime}(r \zeta)\right)=\left[q(r \zeta]^{1-\alpha}\left[q(r \zeta)+m t\left\{h^{1 / \alpha}\left(r \zeta q^{(1-1 / \alpha)}(r \zeta)-q(r \zeta)\right\}\right]^{\alpha}=\right.\right.\right.$

$\left[(1-m r) q^{1 / \alpha}(r \zeta)+m r h^{l / \alpha}(r \zeta) q^{\alpha-1}(r \zeta)\right]^{\alpha} \notin h_{r}(U)$

This completes the proof.

\section{Proof of Theorem 2}

The proof requires the following lemmas and definition:

Lemma 2: [Miller and Mocanu, 1981, Lemma1]. Let $q(z) \in Q$ with $q(0)=a$, and let

$i(z)=a+f_{n} z^{n}+\ldots$, be regular in $U$ with $f(z) \neq a$ and $n \geq 1$. If there exists a point $z_{0} \in U$ such that $f\left(z_{0}\right) \in q(\partial U)$ and $f(/ z /</ z d) \subset q(U)$, then

$$
z_{0} f^{\prime}\left(z_{0}\right)=m \zeta_{0} q^{\prime}\left(\zeta_{0}\right) \text { and }
$$

$$
\operatorname{Re}\left\{1+\frac{z_{0} f^{\prime \prime}\left(z_{0}\right)}{f^{\prime}\left(z_{0}\right)}\right\} \geq m \operatorname{Re}\left\{1+\frac{\zeta_{0} q^{\prime \prime}\left(\zeta_{\phi_{0}}\right)}{\dot{q}^{\prime}\left(\zeta_{0}\right)}\right\}, \text { where } q^{-1}\left(f\left(z_{0}\right)\right)=\zeta_{0}=e^{i \theta_{0} \text { and } m \geq n \geq 1 .}
$$

Lemma 3: [Pommerenke, 1975]. The function $L(z, t)=a_{1}(t) z+\ldots$, with $a_{1}(t) \neq 0 \forall t \geq 0$ is a subordination chain if and only if

$$
\operatorname{Re}\left\{\frac{z \partial L}{\partial z} / \frac{\partial L}{\partial t}\right\}>0 \forall z \in U \text { and } t \geq 0
$$

Definition 2.4: The function $L(z, t), z \in U, t \geq 0$, is a subordination chain if $L(., t)$ is regular and univalent in $U$ for all $t \geq 0$, $L(z,$.$) is continuously differentiable on [0, \infty[\forall z \in U$, and $L(z, s) \prec L(z, t)$, when $0 \leq s \leq t$.

Let $\mathrm{P}(z)=\left(\frac{z p^{\prime}(z)}{p(z)}\right)^{\alpha}$.

Then $P(z)$ is regular in $U$ and $P(0)=1$.

(1.15) can be written as

$$
\mathrm{P}(\mathrm{z})+\frac{\mathrm{z} \mathrm{P}^{\prime}(\mathrm{z})}{\mathrm{P}(\mathrm{z})} \prec h(z)
$$

To prove that $p(z)$ is starlike is equivalent to proving that $P(z) \prec h(z)$ (since it would imply $\operatorname{ReP}(z)=$ $\left.R e\left(\frac{z p^{\prime}(z)}{p(z)}\right)^{\alpha}>0\right)$.

Assume that the functions $\mathrm{P}(z)$ and $h(z)$ satisfy the conditions of the theorem on $\bar{U}$. Else replace $P(z)$ by $P_{r}(z)=$ $\mathrm{P}(r z)$ and $h(z)$ by $\left.h_{r}(z)=h(r z), r \in\right] 0,1\left[\right.$, so that $P_{r}$ and $h_{r}$ satisfy the conditions of the theorem on $\bar{U}$. We would then show that $\left.\mathrm{P}, \prec h_{r} \forall r \in\right] 0,1\left[\right.$ and obtain $\mathrm{P} \prec h$ by letting $r \rightarrow 1^{-}$. 
Case 1: (1.12) and (1.13) are satisfied, but $P(z)$ is not subordinate to $h(z)$. By lemma 2 there exist points $z_{0} \in U$ and $\zeta_{0} \epsilon$ $\partial J$ and an $m \geq 1$ such that $P\left(z_{0}\right)=h\left(\zeta_{0}\right)$ and $z_{0} P^{\prime}\left(z_{0}\right)=m \zeta_{0} h^{\prime}\left(\zeta_{0}\right)$. So for this $z_{0}$,

$$
\begin{gathered}
\mathrm{P}\left(\mathrm{z}_{0}\right)+\frac{\mathrm{Z}_{0} \mathrm{P}^{\prime}\left(\mathrm{z}_{0}\right)}{\mathrm{P}\left(\mathrm{z}_{0}\right)}=h\left(\zeta_{0}\right)+m \frac{\zeta_{0} h^{\prime}\left(\zeta_{0}\right)}{h\left(\zeta_{0}\right)} \\
\arg \left(\frac{\zeta_{0} h^{\prime}\left(\zeta_{0}\right)}{h\left(\zeta_{0}\right)}\right)=\arg \left(\zeta_{0} h^{\prime}\left(\zeta_{0}\right)\right)+\arg \left(h^{-1}\left(\zeta_{0}\right)\right)
\end{gathered}
$$

From (1.12), $\operatorname{Re}\left(h^{-1}\left(\zeta_{0}\right)\right)>0$ and we obtain

$$
\arg \left|\left(h^{-1}\left(\zeta_{0}\right)\right)\right| \leq \frac{\pi}{2}
$$

Aiso $\zeta_{0} h^{\prime}\left(\zeta_{0}\right)$ is an outside normal to the boundary of the convex domain $h(U)$. This together with $(2.10)$ implies that the expression in (2.9) represents a complex outside of $h(U)$. This contradicts (2.8) and we conclude that $P \prec h$.

Case 2: (1.12) and (1.14) are satisfied, then the function

$$
L(z, t)=h(z)+t \frac{z h^{\prime}(z)}{h(z)}=h(z)+t H(z)
$$

is regülar in $U$ for $t \geq 0$.

$$
\frac{\partial L(0, t)}{\partial t}=h^{\prime}(0)[1+t] \neq 0 \text { for } t \geq 0
$$

$L(z, t)$ is also continuously differentiable on $[0, \infty[\forall z \in U$.

$$
\operatorname{Re}\left\{\frac{z \partial L}{\partial z} / \frac{\partial L}{\partial t}\right\}=\operatorname{Reh}(z)+t \operatorname{Re}\left\{\frac{z H^{\prime}(z)}{H(z)}\right\}>0, t \geq 0,
$$

(by (1.12) and (1.14)).

By lemma $3 L(z, t)$ is a subordination chain and we have $L(z, s) \prec L(z, t)$ for $0 \leq s \leq t$.

From (2.11) we obtain

$$
h(z)=L(z, 0) \text {. }
$$

Hence

$$
L(\zeta, t) \notin h(U) \text { for }|\zeta|=1 \text { and } t \geq 0 .
$$

Assume $P(z)$ is not subordinate to $h(z)$. As in case 1 we have

$$
\mathrm{P}\left(\mathrm{z}_{0}\right)+\frac{\mathrm{Z}_{0} \mathrm{P}^{\prime}\left(\mathrm{z}_{0}\right)}{\mathrm{P}\left(\mathrm{z}_{0}\right)}=L\left(\zeta_{0}, m\right), z \in U,\left|\zeta_{0}\right|=1 \text { and } m \geq 1 \text {. }
$$

(2.16) combined with (2.14) contradicts (2.8) and we again conclude that $P \prec h$. This completes the proof

\section{REFERENCES}

Búlboaca T., 2004. Generalised Briot-Bouquet Differential Subordinations and Superordinations, $5^{\text {th }}$. Joint Conference on Math. And Comp. Sci., June 9-12, Debrecen, Humgary ,...

Goodman, A. W., 1983. Univalent Functions (Vol. 1), Mariner Pub., Tampa, Florida. Pp.246 
Kanas, S., 1992. Differential inequalities and differential subordinations. The fourth Finish - Polish Summer School in Complex Analysis and quasi-conformal mappings. Jyvaskyla, Finland, August $17-20: 1-11$.

Miller, S.S. and Mocanu, P.T., 1978. Second Order Differential Inequalities in the Complex plane, J. Math. Anal. Appl. 65(2): $289-305$.

Miller, S.S. and Mocanu, P.T., 1981. Differential subordinations and univalent functions, Michigan Math. J., 28. 157 171.

Miller ,S. S. and Mocanu, P.T., 1985. On some classes of first order differential subordinations, Michigan math. J. 32: $185-195$.

Mfocanu, P.T., 2004. Certain conditions for starlikeness. $5^{\text {th }}$ Joint Conference on Maths and Comp. Sci., June 9 12. Debrecen, Hungary.

Obradovic, M. and Owa, S., 1991. On certain properties for some classes of starlike functions. Journal of Mathematical Analysis and Appl. Vol. 145, $\mathrm{N}^{\circ} 2$.

Owa, S. and Obradovic, M., 1990. An application of differential subordinations and some criteria for univalency. Bull. Austral. Math. Soc., 41: $487-494$.

Pommerenke, C. H., 1975. Univalent functions, Vander hoeck \& Ruprecht, Gottingen. 